\title{
PSYCHOPHYSICAL HEALTH IN RELATION TO THE ENVIRONMENTAL NOISE PERCEPTION IN YOUNGER VERSUS OLDER GROUPS OF PEOPLE IN DHAKA CITY
}

\author{
Kazi Saifuddin*, Md. Shahinuzzaman, Noor Muhammad and Mohammad Abdul Quader ${ }^{1}$ \\ Department of Psychology, Jagannath University, Dhaka 1100, Bangladesh \\ ${ }^{1}$ Department of Geography and Environment, Jagannath University, Dhaka, Bangladesh \\ *Corresponding author; email: kazi_64@yahoo.com
}

\begin{abstract}
Auditory perception towards environmental noise was tested individually in 510 graduate and undergraduate student participants (18-28 yrs) who were experienced in habitually noisy-living areas. A 15-item questionnaire was developed to measure the auditory perception and the responses were evaluated by 10 teachers of Psychology living and working in Dhaka City. Another 33-item 'Burns Anxiety Inventory' questionnaire for the assessment of psychophysical health was used for the same participants. Results showed that increased level of negative perception towards environmental noise maintain a significant linear relation with the degradation of psychophysical health $(\mathrm{P}<0.01)$. Another study similar to the above one was performed in which age factor was tested as a parameter. The results of the second study on 345 older participants (55-65 yrs) showed a significant improvement of psychophysical health $(\mathrm{P}<0.05)$, though the respondents have had negative perception towards environmental noise. The inverse results of these two studies have been interpreted by assuming a threshold function $(\lambda)$ between the two age groups, where $\lambda$ represented a psychophysical antibody against the effects of environmental noise perception.
\end{abstract}

Key words: Psychophysical health, environmental noise perception, psychophysical antibody

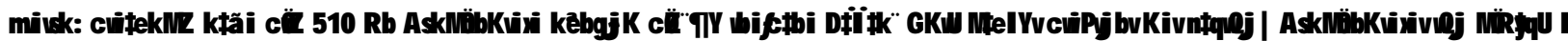

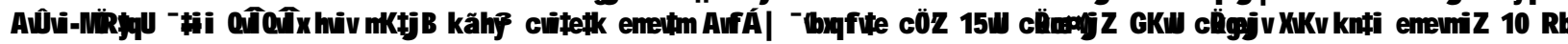

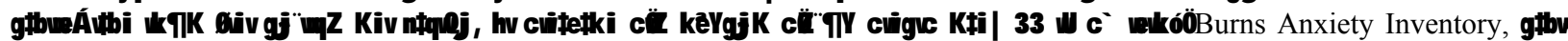

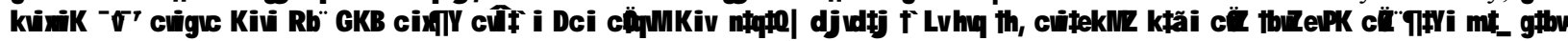

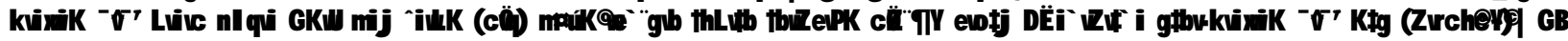

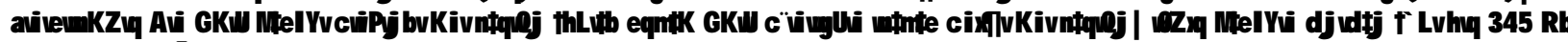

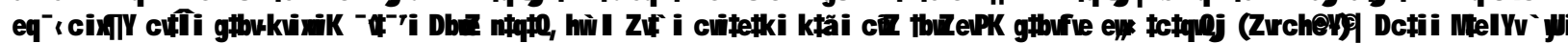

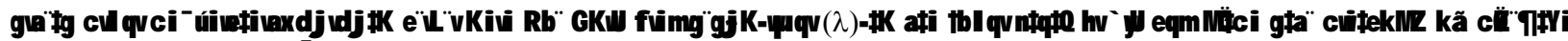

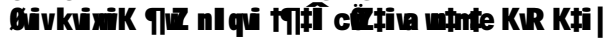

\section{Introduction}

Noise is sound, no doubt, but it can specifically be defined as the unwanted sound. It can also be explained mathematically as the auditory perception of the randomized-wave form of sound. In another way, noise has been defined as the level of sound which exceeds the acceptable level and creates annoyance (Alam et al. 2000). The word noise is derived from the Latin word nausea meaning sickness. Noise is, however, among the most pervasive pollutant today. Everyday noises in the urban areas from road traffic, jet planes, construction equipment and manufacturing processes, to name a few, are the unwanted sounds that are routinely broadcast into the air, and these have been called environmental noise (Saifuddin et al. 2001, Saifuddin 2002a).

Dhaka is one of the biggest metropolitans and one of the most polluted cities in the world. Like air and water pollution, the inhabitants of this city are being exposed to high levels of noise pollution (Ahmed 1998, Alam et al. 2000). The noise pollution has been evaluated here on the basis of such levels that correspond to psychophysical health (Lundberg and Frankenhaeuser 1978, Saifuddin 2002b). Such sound levels are mostly dependent on the intensity and pressure. The intensity meaning as the power-density spectrum is the average rate of sound energy transmitted through a unit area perpendicular to the direction of sound propagation, typically measured in Pico-watts per square metre. Sound pressure is usually proportional to the square root of sound power. Regarding this, a logarithmic measure called decibel $(\mathrm{dB})$ is used to describe sound-pressure level. The A-weighted sound level, measured in decibel (dBA), is the generally accepted scale for measuring sound level in highway transportation. However, studies on the acceptable levels of noises during day and night times in different areas of Dhaka City (Table 1) and noise levels at 37 roadside locations in Dhaka City during a 24$\mathrm{hr}$ period (Table 2) reveal some interesting findings. 
From his studies, Ahmed (1998) established a linear relationship between the noise levels and the traffic volume as expressed by the equation: Sound level $(\mathrm{dBA})=43.818+0.0044 T \mathrm{~T} ;(\mathrm{R} 2=0.98)$ where, $T \mathrm{~V}$ denotes traffic volume in passenger car unit.

In a study conducted in Osaka City, Japan, the incidence of low-birth weight babies was found to be increased as the noise level of mother's living area increased, suggesting that such noise could be a possible cause of toxemia during pregnancy (Ando and Hattori 1974). In another study on the inhabitants around the Osaka International Air Port, Ando and Hattori (1977) found that the human placental lactogen levels in pregnant mothers after the 30th week of pregnancy in the noisy areas tended to be lower than those in the reference areas. Iwata (1984) conducted an experiment on the relationship of noise sensitivity to health and personality, and he noted that high noise sensitivity is more associated with mental symptoms than physical ones. In subsequent studies (Ando 2000; Saifuddin 2003) human auditory sensation has been designated as the primary auditory sensation. Keeping the above findings in mind, here an attempt has been made to assess the psychophysical health $(\mathrm{PH})$ in relation to environmental noise perception in two age groups of subjects. Then, depending on the results, a hypothesis has been tested in which human auditory perception towards environmental noise pollution is related to the psychophysical health. Therefore aim of this study was to investigate the relationship between the perceptual phenomena of the inhabitance towards the environmental noise and their psychophysical health status. Noise perception as an individual cognition towards the physical-environmental exposure psychophysical health can be effected individually through internal physiological correlates. The age factor as individualistic characteristics can also be a parameter between such perceptual process and the psychophysical health status. As it is well known that the age factor is the key determinant of perception as well as the health status to the age effects in this regard was another aim of this study.

Table 1. Noise levels as standard in different areas of Dhaka City (After Ahmed 1998).

\begin{tabular}{lll}
\hline Description of Areas & $\begin{array}{l}\text { Noise levels (dBA) } \\
\text { Day time Night time }\end{array}$ \\
\hline Hospital, School, Mosque etc. & 50 & 40 \\
Residential area & 55 & 45 \\
Mixed area & 60 & 50 \\
Commercial area & 70 & 60 \\
Industrial area & 75 & 70 \\
dBA= Decibel A-weighting & &
\end{tabular}

Table 2. Roadside levels of noises (in dBA) in different areas of Dhaka City, measured during a 24 hr period (After Alam et al. 2000).

\begin{tabular}{cccccccc}
\hline $\begin{array}{c}\text { Time } \\
\text { Intervals }\end{array}$ & \multicolumn{9}{c}{ Dhanmondi } & $\begin{array}{l}\text { Farm- } \\
\text { gate }\end{array}$ & Gulistan & Gulshan & Syadabad & $\begin{array}{l}\text { Science } \\
\text { Lab. }\end{array}$ & Uttara \\
\hline Area type & $\mathrm{R}$ & $\mathrm{C}$ & $\mathrm{C}$ & $\mathrm{R}$ & $\mathrm{C}$ & $\mathrm{M}$ & $\mathrm{R}$ \\
$7 \mathrm{am}-11 \mathrm{am}$ & 75.89 & 80.07 & 80.08 & 76.16 & 83.27 & 76.24 & 76.25 \\
$11 \mathrm{am}-3 \mathrm{pm}$ & 74.38 & 78.86 & 79.34 & 74.83 & 83.89 & 75.19 & 74.81 \\
3pm-7pm & 75.21 & 81.96 & 81.13 & 76.11 & 84.37 & 77.23 & 76.81 \\
$7 \mathrm{pm}-11 \mathrm{am}$ & 76.30 & 80.28 & 78.52 & 74.31 & 82.08 & 75.32 & 73.36 \\
Average & 75.44 & 80.29 & 79.77 & 75.35 & 83.40 & 76.00 & 75.31 \\
\hline R= Residential; $\mathrm{C}=$ Commercial; $\mathrm{M}=$ Mixed & & & & &
\end{tabular}

Residential; $\mathrm{C}=$ Commercial; $\mathrm{M}=$ Mixed.

\section{Materials and Methods}

Participants: Graduate and undergraduate students of both sexes $(\mathrm{N}=510)$, aged between 18 and 28 years, took part in the study as younger participants. In addition, 345 professionals belonging to 55 to 65 years of age were used as older participants. All of subjects were selected at random, sometimes using personal choices to those who were habitually living in the high- noisy residential areas of Dhaka City. The participants were interviewed individually without any formalities, but all of them were previously informed (prepared) for interview. All the respondents had good educational background, they were all conscious about noise pollution, and were of normal hearing.

Perception-measuring questionnaire: A questionnaire written in Bengali was developed to assess the 
perception of the subjects towards noise pollution. A set containing 25 questions was selected primarily by the research group and then it was sent to 10 teachers of Psychology living and working in Dhaka City. The teachers scrutinized the questions on the basis of their professional experiences and judgments. Thus, the questions were re-evaluated and finally 15 questions (items) were chosen for the study. First 12 items in the set had the following four options for showing responses: $0=$ not at all, $1=$ a few, $2=$ average and $3=$ too much. Last three items in the set contained individually three and more options to be answered. All the answers were recorded by using tick mark $(\sqrt{ })$ on the questionnaire.

Health-measuring questionnaire: Burns-Anxiety Inventory (BAI), devised by Burns (1990), was used to evaluate psychophysical health $(\mathrm{PH})$ of the individuals. The BAI contains 33 items and four options $(0,1,2,3)$ to answer in Bengali. The BAI was also divided into three categories in which Category 1 assessed anxious feelings; Category 2 assessed anxious thoughts; and Category 3 assessed physical symptoms. Total scores recorded for each individual were analyzed using BAI as shown in Table 3.

Table 3. Scoring and problem identifying keys of the Burns Anxiety Inventory (after Burns 1990).

$\begin{array}{cc}\text { No. } & \text { Score range } \\ 1 & 0-4 \\ 2 & 5-10 \\ 3 & 11-20 \\ 4 & 21-30 \\ 5 & 31-50 \\ 6 & 51-99\end{array}$

Nature of problems Minimal or no anxiety Borderline anxiety Mild anxiety Moderate anxiety Severe anxiety Extreme anxiety with physical symptoms

Procedures: Younger students and older professionals were selected through personal correspondence and were interviewed individually. The interviews took part either in the Department or in the residence of the participants. The subjects were explained primarily about the importance of the interview. The interviewers asked the subjects directly and provided specific marks according to the individual answers. The experimental data were finally scored by using averaging method and for determination test of significance were also used.

\section{Results and Discussion}

Noise perception in younger respondents: Results on the first study (Table 4; Fig. 1) demonstrate that increased negative perception (NP) towards noise pollution was linearly related $(\mathrm{P}<0.01)$ to psychophysical health degradation (HD), which could be shown by the equation: $\mathrm{y} \approx \mathrm{mx}$ in which $\mathrm{y}, \mathrm{x}$ and $\mathrm{m}$ indicate the HD, NP and a linear function, respectively.
Table 4. Psychophysical health scores according to age groups and noise perception (NP) in young (18-28 yrs) and older (55$65 \mathrm{yrs})$ participants.

\begin{tabular}{|c|c|c|c|c|}
\hline $\begin{array}{c}\text { Participants (Age } \\
\text { groups in yrs) }\end{array}$ & $\begin{array}{c}\text { Scores } \\
\text { on NP }\end{array}$ & $\begin{array}{c}\text { Mean } \\
\text { PPH }\end{array}$ & Number & SD \\
\hline Younger & 29.40 & 91.06 & 14.00 & 4.14 \\
$18-21$ & 33.60 & 91.79 & 52.60 & 5.20 \\
$22-25$ & 33.60 & 94.07 & 21.40 & 3.98 \\
$26-28$ & & & $\sum \mathrm{N}=88.0$ & \\
& & & & \\
Older & 28.64 & 25.33 & 06.86 & 2.03 \\
$55-58$ & 31.40 & 18.59 & 13.80 & 3.69 \\
$59-62$ & 26.50 & 20.16 & 07.00 & 5.09 \\
$63-65$ & & & $\sum \mathrm{N}=27.66$ & \\
\hline
\end{tabular}

$\mathrm{PPH}=$ Psychophysical health; $\mathrm{SD}=$ Standard deviation,

Noise perception in older respondents: Negative perception towards noise pollution in the second study was found to have an inverse relation $(\mathrm{P}<0.05)$ with the HD (Table 4; Fig. 2), meaning that if the perception is negative towards noise, psychophysical health improved. Results of the two studies, therefore, are controversial owing only to differences in the age parameter of the respondents.

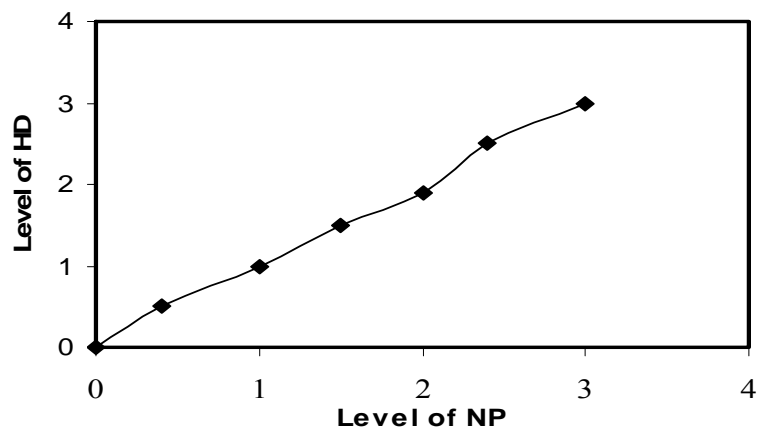

Fig. 1. Negative perception (NP as scale value) towards noise pollution in relation to psychophysical health degradation (HD) in younger respondents (18-28 yrs' age-groups).

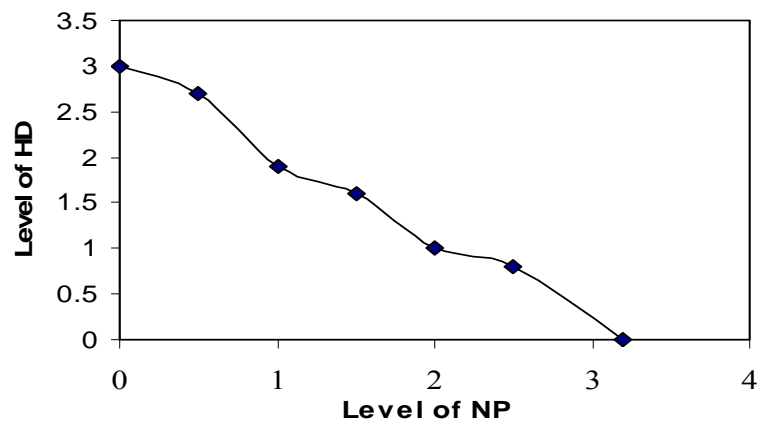

Fig. 2. Negative perception (NP as scale value) towards noise pollution in relation to psychophysical health degradation (HD) in older respondents (55-65 yrs' age groups). 


\section{Conclusion}

The present controversial results on HD in terms of environmental noise perception (NP) can be explained by an internal threshold mechanism which may be developed in between older and young age. The threshold mechanism (TM) might be accelerated by auditory-temporal perception, which is found from the significant experiment conducted by Saifuddin (2001b). The TM as a function achieved by experience as the threshold function $(\lambda)$ which, can be controlled by hypothalamus of the brain in which, sympathetic and para-sympathetic systems are involved. If the individual has been living in environmentally noisy area for a long time, such TM can be developed. That is why, in spite of chronic environmental noise, the psychophysical health might be improved. The proposed threshold function, however, may be interpreted here as a psychophysical antibody to explain the observed controversy.

\section{References}

Ahmed K. 1998. A Study on Noise Pollution in Dhaka City. Directorate of Environment, The Government of the People's Republic of Bangladesh.

Alam JB, Hoque MM and Ahmed MF. 2000. Traffic induced noise pollution in Dhaka City. Bangladesh Environment 2000, 77-83.

Ando Y. 2000. A theory of primary sensations and perceptions. J. Sound Vibration 241: 3-18.
Ando Y and Hattori H. 1974. Reaction of infants to air-craft noise and effects of the noise on human-fetal life. Practica Otologica Kyoto 67: 129-136.

Ando Y and Hattori H. 1977. Effects of noise on humanplancental lactogen levels in maternal plasma. British J. Obstetrics Gynecol. 84: 115-118.

Burns DD. 1990. Burns Anxiety Inventory. Feeling Good Handbook, Plume, North Austin (Last modification: April 22, 2003).

Iwata O. 1980. Determinants of noise effects. In: Noise and Vibration Research Group of Environmental Sciences. Japanese Ministry of Education Grant-in-Aid for Scientific Research.

Iwata O. 1984. The relationship of noise sensitivity to health and personality. Japanese Psychol. Res. 26(2): 75-81.

Lundberg U and Frankenhaeuser M. 1978. Psycho-physiological reactions to noise as modified by personal control over noise intensity. Biol. Psychol. 6: 51-59.

Saifuddin K. 2001. Relationship between auditory-evoked potential and subjective duration in respect of sound pressure level. Bangladesh Psychol. Studies 11: 69-76.

Saifuddin K. 2002a. Four dimensional standards of traffic noises in Dhaka City. Bangladesh Environment 1: 128-133.

Saifuddin K. 2002b. Differential thresholds of subjective durations of bandpass noises and complex noises. Bangladesh Psychol. Studies 12: 37-42.

Saifuddin K. 2003. A theory of sensory dimension of auditory process. Bangladesh Psychol. Studies 13: 37-46.

Saifuddin K, Sakai H and Ando Y. 2001. Duration sensation of simulated environmental noise in relation to its autocorrelation function. J. Sound Vibration 241: 117-127.

Manuscript received on 21 December and revised on 17 January 2001. 